JYFL-8/98

US-FT/14-98

hep-ph/9807297

July 1998

\title{
THE SCALE DEPENDENT NUCLEAR EFFECTS IN PARTON DISTRIBUTIONS FOR PRACTICAL APPLICATIONS
}

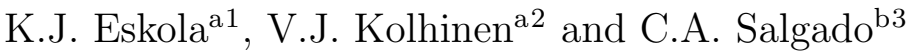 \\ a Department of Physics, University of Jyväskylä, \\ P.O.Box 35, FIN-40351 Jyväskylä, Finland \\ b Departamento de Física de Partículas, \\ Universidade de Santiago de Compostela, \\ E-15706 Santiago de Compostela, Spain.
}

\begin{abstract}
The scale dependence of the ratios of parton distributions in a proton of a nucleus $A$ and in the free proton, $R_{i}^{A}\left(x, Q^{2}\right)=f_{i / A}\left(x, Q^{2}\right) / f_{i}\left(x, Q^{2}\right)$, is studied within the framework of the lowest order leading-twist DGLAP evolution. By evolving the initial nuclear distributions obtained with the GRV-LO and CTEQ4L sets at a scale $Q_{0}^{2}$, we show that the ratios $R_{i}^{A}\left(x, Q^{2}\right)$ are only moderately sensitive to the choice of a specific modern set of free parton distributions. We propose that to a good first approximation, this parton distribution setdependence of the nuclear ratios $R_{i}^{A}\left(x, Q^{2}\right)$ can be neglected in practical applications. With this result, we offer a numerical parametrization of $R_{i}^{A}\left(x, Q^{2}\right)$ for all parton flavours $i$ in any $A>2$, and at any $10^{-6} \leq x \leq 1$ and any $Q^{2} \geq 2.25 \mathrm{GeV}^{2}$ for computing cross sections of hard processes in nuclear collisions.
\end{abstract}

\footnotetext{
${ }^{1}$ kari.eskola@phys.jyu.fi

${ }^{2}$ vesa.kolhinen@phys.jyu.fi

${ }^{3}$ salgado@fpaxp1.usc.es
} 


\section{Introduction}

The measurements of the nuclear structure function $F_{2}^{A}\left(x, Q^{2}\right)$ in deeply inelastic leptonnucleus scattering (DIS) [1]-[9] indicate clearly that parton distributions of the bound protons are different from those of the free protons, $f_{i / A}\left(x, Q^{2}\right) \neq f_{i / p}\left(x, Q^{2}\right)$. The nuclear effects are often categorized according to those observed in the ratio of the structure functions of nuclei relative to deuterium, $R_{F_{2}}^{A} \equiv F_{2}^{A} / F_{2}^{\mathrm{D}}$ : shadowing $\left(R_{F_{2}}^{A} \leq 1\right)$ at Bjorken- $x \lesssim 0.1$, anti-shadowing $\left(R_{F_{2}}^{A} \geq 1\right)$ at $0.1 \lesssim x \lesssim 0.3$, EMC effect $\left(R_{F_{2}}^{A} \leq 1\right)$ at $0.3 \lesssim x \lesssim 0.7$, and Fermi motion $\left(R_{F_{2}}^{A} \geq 1\right)$ towards $x \rightarrow 1$ and beyond. The recent high-precision measurements by the New Muon Collaboration (NMC) of the structure function $F_{2}$ of tin vs. that of carbon, $F_{2}^{\mathrm{Sn}} / F_{2}^{\mathrm{C}}$ [8], have also revealed a $Q^{2}$-dependence at small values of $x$.

Theoretically, the origin of the nuclear effects is still under debate but it is believed that different mechanisms are responsible for the effects in the different regions of $x$. For a compact introduction and references to the various theoretical models we refer the reader to Arneodo's review, Ref. [9].

In this paper, as a sequel to Ref. [10], we are going to focus on studying the perturbative QCD-evolution of the ratios $R_{i}^{A} \equiv f_{i / A}\left(x, Q^{2}\right) / f_{i / p}\left(x, Q^{2}\right)$ within the framework of lowest order (LO) leading twist (LT) DGLAP evolution [11]. To our knowledge the perturbative QCD-evolution of nuclear parton densities has been studied at least in Refs. [12]- [22].

Our approach is in practice very similar to the case of the free proton: once the absolute nuclear parton distributions are given at an initial scale $Q^{2}=Q_{0}^{2} \gg \Lambda_{\mathrm{QCD}}^{2}$ and at some $x_{\text {min }} \leq x \leq 1$, the further evolution in $Q^{2}$ is predicted (at this range of $x$ ) by the DGLAP equations. In other words, as in [10], we assume that at $Q^{2} \gtrsim Q_{0}^{2}$ the scale evolution of the nuclear parton densities is purely perturbative and that the initial conditions at $Q_{0}^{2}$ contain nonperturbative input. We determine the initial nuclear parton distributions iteratively through the QCD evolution by using experimental data and conservation of momentum and baryon number as constraints. It should be noted that we do not try to explain the origin of the nuclear effects but we study their behaviour once they are there at an initial scale. Furthermore, since we neglect all the higher twist contributions, like the perturbative GLRMQ terms 23] at small values of $x$, no nuclear effects are generated through the evolution but they are all hidden in the initial distributions at $Q_{0}^{2}$.

In Ref. [10], the DIS data on $F_{2}^{A} / F_{2}^{\mathrm{D}}$ and $F_{2}^{A} / F_{2}^{\mathrm{C}}$ [2]-[7] were used together with the measurements of the Drell-Yan (DY) cross sections in $p A$ vs. $p \mathrm{D}$ collisions [24, and with the conservation of baryon number and total momentum to determine the initial nuclear parton distributions at a scale $Q_{0}^{2}=2.25 \mathrm{GeV}^{2}$. Especially, it was shown in 10 that the LT LO DGLAP-evolution can account very well for the $Q^{2}$ dependence observed in the experimental ratio $F_{2}^{\mathrm{Sn}} / F_{2}^{\mathrm{C}}[8$.

The measurements of the ratios $R_{F_{2}}^{A}$ in DIS, and also the ratios of nuclear Drell-Yan (DY) cross sections relative to deuterium, provide us with information on the nuclear parton distributions only relative to the nucleons in deuterium. In an approximation where the small nuclear effects in deuterium are neglected, as done in [10] and as we will do here as well, we get information relative to the free nucleons. Then, quite obviously the extraction of the absolute nuclear parton distributions depend on our choice for the set of parton distributions in the 
free proton, and the QCD-evolution itself will depend on this choice, toof. In Ref. [10], it was anticipated that the ratios $R_{i}^{A}\left(x, Q^{2}\right)$ should not depend as strongly on the choice for the set as the absolute distributions do. In this paper, we will verify this statement quantitatively by comparing the scale-evolved nuclear effects initially obtained by using the GRV-LO [25] and CTEQ4L [26] distributions.

After demonstrating that the set-dependence in the nuclear ratios $R_{i}^{A}\left(x, Q^{2}\right)$ for individual flavours indeed is a negligible effect as compared to the current overall uncertainties, it becomes more meaningful to present a numerical parametrization of $R_{i}^{A}\left(x, Q^{2}\right)$ for practical applications. The parametrization can then be used with any modern set of LO parton distributions to obtain the absolute nuclear parton distributions needed for computing hard scattering cross sections in nuclear collisions. The parametrization we offer can be called for any parton flavour $i$, any $A>2,10^{-6} \leq x \leq 1$ and $Q^{2} \geq 2.25 \mathrm{GeV}^{2}$. The parametrization, which we give in form of a short Fortran code, is now available from us?.

\section{The framework and the assumptions}

A detailed formulation of our approach can be found in Ref. [10 but let us recall the underlying basic assumptions also here. The starting point is the measured ratio of structure functions of a nucleus $A$ and deuterium $\mathrm{D}, R_{F_{2}}^{A} \equiv F_{2}^{A} / F_{2}^{\mathrm{D}}$. In the one-photon approximation the ratio of the cross sections measured in $l A$ and in $l \mathrm{D}$ gives directly the ratio $R_{F_{2}}^{A}$, provided that the ratio of the cross sections of longitudinally and transversally polarized virtual photons with the target, $R \equiv \sigma_{L} / \sigma_{T}$, does not depend on the target. So far, experiments have not shown any significant target dependence [9, 4, 8]. Our approach, being currently only in the lowest order in the cross sections, will not generate such a dependence, either.

First, the structure function ratio $R_{F_{2}}^{A} \equiv F_{2}^{A} / F_{2}^{\mathrm{D}}$ is expressed in terms of nuclear parton distributions by using the LO relation in the QCD improved parton model, $F_{2}=$ $\sum_{q} e_{q}^{2}\left[x f_{q}\left(x, Q^{2}\right)+x f_{\bar{q}}\left(x, Q^{2}\right)\right]$. By the nuclear distributions $f_{i / A}$ of a parton flavour $i$ we mean the average distributions of a parton flavour $i$ in a proton of a nucleus $A$ : $f_{i / p / A}\left(x, Q^{2}\right) \equiv$ $f_{i / A}\left(x, Q^{2}\right)$. In principle, the nuclear parton distributions are non-zero up to $x \rightarrow A$ but the small tails are completely negligible from the point of view of our study. We therefore approximate $f_{i / A}\left(x \geq 1, Q^{2}\right)=0$.

For isoscalar nuclei $d_{n / A}=u_{p / A} \equiv u_{A}$ and $u_{n / A}=d_{p / A} \equiv d_{A}$ obviously hold but we have to assume that these are good approximations for non-isoscalar nuclei as well 5 . We also neglect the small nuclear effects in deuterium [27]. With these approximations $R_{F_{2}}^{A}$ reflects directly the deviations of $F_{2}^{A}$ from the free nucleons.

The nuclear effects in the parton distributions are defined through the ratios for each flavour as,

$$
R_{i}^{A}\left(x, Q^{2}\right) \equiv \frac{f_{i / A}\left(x, Q^{2}\right)}{f_{i}\left(x, Q^{2}\right)}
$$

\footnotetext{
${ }^{4}$ We will refer to the dependence on the choice for the set of parton distributions in the free proton as "set-dependence"

${ }^{5}$ via email or in the WWW, http://fpaxp1.usc.es/phenom/

${ }^{6}$ For non-isoscalar nuclei the corresponding exact relation is $d(u)_{n / A_{Z}}=u(d)_{p / A_{A-Z}}$
} 
where $f_{i}$ is the distribution of the flavour $i$ in the free proton as given by the chosen set of parton distributions. The modern sets include the rapid increase of the gluon and sea quark distributions at small values of $x$, in accordance with the rise discovered in the structure function $F_{2}^{e p}$ at HERA [28]. In the DGLAP evolution we treat the massive quarks as massless and generate them only radiatively above fixed threshold scales. The two modern LO sets of parton distributions in which the heavy quarks are treated in this manner are the GRV-LO [25] and CTEQ4L [26], which is why we use only these two sets in this work.

To eliminate the complication of determining nuclear effects for the heavy quark distributions at the initial scale $Q_{0}^{2}$, we choose $Q_{0}^{2}$ at or below the charm-threshold $Q_{c}^{2}$, where $x c=x c_{A}=0$. In the set GRV-LO the threshold is $Q_{c}^{2}=2.25 \mathrm{GeV}^{2}$ and in the CTEQ4L $Q_{c}^{2}=2.56 \mathrm{GeV}^{2}$. To be consistent, we use these in the corresponding evolution in the nuclear case as well. The HERA results have also shown that the leading twist evolution describes the structure function $F_{2}^{e p}$ well at $Q^{2} \gtrsim 1 \mathrm{GeV}^{2}$ and $x \gtrsim 10^{-4}$ [29]. In the nucleus, however, the higher twist effects can be expected to be stronger [23, 12, 16]. Since we will not include these here, we should not start the perturbative evolution of nuclear parton distributions below $Q^{2} \lesssim 1 \mathrm{GeV}^{2}$. Therefore, for not too small values of $x$, it is fairly safe to choose $Q_{0}^{2}=Q_{c}^{2}=2.25 \mathrm{GeV}^{2}$ of the set GRV-LO. The results in Ref. [10] show that this is a valid initial scale at least for $x \gtrsim 0.01$, where the higher twist contributions are negligible.

As further approximations, but for the initial conditions at $Q^{2}=Q_{0}^{2}$ only, it is assumed that the differencies between the nuclear effects for sea quarks and antiquarks, as well as for different sea quark flavours are negligible: $R_{\bar{q}}^{A}\left(x, Q_{0}^{2}\right)=R_{q_{\mathrm{sea}}}^{A}\left(x, Q_{0}^{2}\right)=R_{S}^{A}\left(x, Q_{0}^{2}\right)$. Similarly, the same nuclear effects are assumed for the valence quarks at the initial scale, $R_{u_{V}}^{A}\left(x, Q_{0}^{2}\right)=$ $R_{d_{V}}^{A}\left(x, Q_{0}^{2}\right)=R_{V}^{A}\left(x, Q_{0}^{2}\right)$. The fact that with these approximations we get a stable evolution - i.e. that the nuclear effects do not rapidly evolve away from what is assumed at $Q_{0}^{2}-$ shows that these are indeed reasonable first approximations.

Then, we arrive at a simple formula for an isoscalar nucleus,

$$
R_{F_{2}}^{A}\left(x, Q_{0}^{2}\right)=A_{V}^{I S}\left(x, Q_{0}^{2}\right) R_{V}^{A}\left(x, Q_{0}^{2}\right)+\left[A_{u d}^{I S}\left(x, Q_{0}^{2}\right)+A_{s}\left(x, Q_{0}^{2}\right)\right] R_{S}^{A}\left(x, Q_{0}^{2}\right),
$$

where the coefficients are known exactly [10]:

$$
\begin{aligned}
A_{V}^{I S}\left(x, Q^{2}\right) & =5\left[u_{V}\left(x, Q^{2}\right)+d_{V}\left(x, Q^{2}\right)\right] / N_{F_{2}}\left(x, Q^{2}\right) \\
A_{u d}^{I S}\left(x, Q^{2}\right) & =10\left[\bar{u}\left(x, Q^{2}\right)+\bar{d}\left(x, Q^{2}\right)\right] / N_{F_{2}}\left(x, Q^{2}\right) \\
A_{s}\left(x, Q^{2}\right) & =4 s\left(x, Q^{2}\right) / N_{F_{2}}\left(x, Q^{2}\right) \\
N_{F_{2}}\left(x, Q^{2}\right) & =5\left[u_{V}\left(x, Q^{2}\right)+d_{V}\left(x, Q^{2}\right)\right]+10\left[\bar{u}\left(x, Q^{2}\right)+\bar{d}\left(x, Q^{2}\right)\right]+4 s\left(x, Q^{2}\right) .
\end{aligned}
$$

They obviously depend on the specific set of parton distributions chosen for the free proton. The above Eq. (2) correlates the nuclear effects of the valence quarks with those of the sea quarks through the ratio $R_{F_{2}}^{A}$. As described in detail in [10], we fix $R_{F_{2}}^{A}$ iteratively through the evolution by using the DIS data [2]-[7], decompose $R_{F_{2}}^{A}$ into $R_{V}^{A}$ and $R_{S}^{A}$ at $Q^{2}=Q_{0}^{2}$ according to Eq. (2) by simultaneously constraining $R_{V}^{A}$ by conservation of baryon number and $R_{S}^{A}$ by the DY data [24]. At large values of $x$ the sea quark distributions are very small as compared to the valence distributions, and the contribution from the sea to $R_{F_{2}}^{A}$ is negligible. This is why we have no constraints for $R_{S}^{A}$ at $x \gtrsim 0.3$. We simply assume that the sea quarks show a similar EMC effect as the valence quarks do, so at large values of $x$ we have $R_{V}^{A}=R_{S}^{A}=R_{F_{2}}^{A}$. 
Also the region below $x \lesssim 0.01$ is not well constrained: there exist experimental DIS data, even at much lower values of $x$ [3, 6], but due to the correlation of $x$ and $\left\langle Q^{2}\right\rangle$ in the experiments, the values of $\left\langle Q^{2}\right\rangle$ are in the nonperturbative region from our point of view. Also, as pointed out in [10], since $\partial R_{F_{2}}^{A}\left(x, Q^{2}\right) / \partial \log Q^{2} \sim R_{G}^{A}\left(2 x, Q^{2}\right)-R_{F_{2}}^{A}\left(x, Q^{2}\right)$, the slope of $R_{F_{2}}^{A}$ in $Q^{2}$ - even the initial sign of the slope - depends on what is assumed for the initial nuclear gluon profile $R_{G}^{A}\left(x, Q_{0}^{2}\right)$ at small values of $x$. We assume a saturation of shadowing in $R_{F_{2}}^{A}$, as observed in the nonperturbative region [3, 6], and again, to reach a stable evolution [10], take $R_{G}^{A}=R_{F_{2}}^{A}$ at small values of $x$. In relation to the data on $R_{F_{2}}^{A}$ measured in the region of very small $x$ [3, 6], we basically expect that the nonperturbative evolution does not change the sign of the $Q^{2}$-slope of $R_{F_{2}}^{A}$. This interesting question is related to the origin of nuclear shadowing. Within our approach, however, we cannot address this problem further.

Apart from the overall momentum conservation, we do not, unfortunately, have any direct constraints for the nuclear gluon distributions. In principle, as pointed out in [14], the $Q^{2}$ dependence of $R_{F_{2}}^{A}$, if measured very accurately at small enough values of $x$, would serve as such a constraint. As a step towards this direction, the NMC has recently measured the $Q^{2}$ dependence of $F_{2}^{\mathrm{Sn}} / F_{2}^{\mathrm{C}}$ [8] and Pirner and Gousset [30] have used this data [31] to extract the approximate nuclear gluon densities. Since we now assume a similar shadowing in $R_{G}^{A}$ as in $R_{F_{2}}^{A}$, we are bound to have some antishadowing due to the overall momentum conservation [10]. In our analysis, we use the results of Ref. [30], to constrain only the point where $R_{G}^{A}\left(x, \widehat{Q_{0}^{2}}\right) \approx 1$. By requiring again a stable evolution (unlike in [14]) we expect that the gluons have an EMC-effect as well. The height of the antishadowing peak in $R_{G}^{A}\left(x, Q_{0}^{2}\right)$ is then fixed by the momentum conservation. As shown in [10], with the full scale evolution this results in quite a good overall agreement with the analysis in Ref. 30.

\section{The set-dependence of the nuclear effects}

In this section, we will make a quantitative comparison of the set-dependence of the nuclear effects for all parton flavours. More exactly, we will compare the results of [10] obtained with the parton distribution set GRV-LO [25] to those obtained with the set CTEQ4L[26].

First, the set-dependence will obviously be the largest for largest nuclei, so it is sufficient to focus on the set-dependence for an isoscalar $A=208$. To have a consistent but the most straightforward comparison, we fix the parametrization of $R_{F_{2}}^{A}\left(x, Q_{0}^{2}\right)$ to be exactly the same as given in [10]. By doing this, we anticipate that the set-dependence of the scale evolution of $R_{F_{2}}^{A}$ will be negligible, so that a new iteration with a new initial parametrization of $R_{F_{2}}^{A}\left(x, Q_{0}^{2}\right)$ is not needed. Similarly, we keep the parameters for $R_{V}^{A}\left(x, Q_{0}^{2}\right)$ at $x \lesssim 0.1$ the same as given in $10 \|$.

To apply the initial parametrization of $R_{F_{2}}^{A}\left(x, Q_{0}^{2}\right)$ to the CTEQ4L distributions in a consistent manner, and to remove a source of uncertainty, we first evolve the CTEQ4L distributions downwards from $Q^{2}=Q_{0}^{2, \mathrm{CTEQ} 4 \mathrm{~L}}=2.56 \mathrm{GeV}^{2}$ to our starting scale $Q^{2}=Q_{0}^{2}=$ $2.25 \mathrm{GeV}^{2}$, and only then apply the procedure of extracting the initial nuclear effects in the valence quarks, $R_{V}^{A}\left(x, Q_{0}^{2}\right)$, in the sea quarks, $R_{S}^{A}\left(x, Q_{0}^{2}\right)$, and in the glue, $R_{G}^{A}\left(x, Q_{0}^{2}\right)$. These initial ratios can be found in Fig. 1, together with the previous results [10 with the set GRV-LO. The resulting set-dependence in $R_{S}^{A}\left(x, Q_{0}^{2}\right)$ and $R_{V}^{A}\left(x, Q_{0}^{2}\right)$ is small and certainly well within the expected overall uncertainty in fixing the initial conditions for these ratios. 
Notice also that the set-dependence of the initial gluon ratio $R_{G}^{A}\left(x, Q_{0}^{2}\right)$ is conveniently small, as seen in Fig. 1, even though the absolute gluon distributions of GRV-LO and CTEQ4L differ from each other, even by a factor $\sim 2$. Remember that the shadowing of $R_{G}^{A}\left(x, Q_{0}^{2}\right)$ at very small values of $x$ was fixed to coincide with $R_{F_{2}}^{A}\left(x, Q_{0}\right)$ but the antishadowing peak in $R_{G}^{A}\left(x, Q_{0}^{2}\right)$ is determined from the overall momentum conservation.

Next, we perform the DGLAP evolution [11] for the absolute nuclear parton distributions now obtained by using the set CTEQ4L, and form the nuclear ratios of individual ratios at a large scale $Q^{2}=10000 \mathrm{GeV}^{2}$. The comparison with the ratios obtained by the set GRV-LO is shown in Fig. 1. It is quite interesting to notice that even if the absolute distributions may differ from each other considerably, in the scale-evolved nuclear ratios the differencies tend to cancel out, making the set-dependence a small effect. The set-dependence is the largest for the gluon ratios at small values of $x$, and this is transmitted directly to the sea quarks and to the heavy quarks. The strange-quarks also show a set-dependence of at most $5 \%$. Another interesting point is that the difference between $R_{\bar{u}}^{A}$ and $R_{\bar{d}}^{A}$ stays quite small even though $\bar{u} \neq \bar{d}$ for the set CTEQ4L.

Bearing in mind the uncertainties in fixing the initial ratios, especially the assumptions regarding shadowing at $x \lesssim 0.01$, and the gluon profile in general, the conclusion is that the set-dependence is a negligible effect as compared to the current overall uncertainty in determining the initial conditions.

In Fig. 2, the set-dependence of the nuclear ratios is shown as function of nuclear mass $A$ at different fixed values of $x$ and one fixed value of $Q^{2}$. This figure demonstrates the fact that for smaller nuclei the set-dependence is even a smaller effect.

\section{The parametrization for practical applications}

Originally [14, 10], we have stored the $x$ - and $Q^{2}$-dependent nuclear ratios, $R_{i}^{A}\left(x, Q^{2}\right)$ for all relevant parton flavours in a big table for each nucleus separately. In practical applications of computing cross sections of hard scatterings in nuclear collisions, these big tables have to be interpolated to obtain the absolute nuclear distributions $f_{i / A}\left(x, Q^{2}\right)=R_{i}^{A}\left(x, Q^{2}\right) f_{i}\left(x, Q^{2}\right)$. Then, in principle, one should store $\sim 200$ tables to be able to get nuclear parton distributions in an arbitrary nucleus $A \geq 2$. To avoid this complication, and to make the nuclear effects more easily available for any user, we have prepared a numerical Fortran code which parametrizes the $x_{-}, Q^{2}$ - and especially the $A$-dependence of $R_{i}^{A}\left(x, Q^{2}\right)$ for all parton flavours $i$ of an isoscalar nucleus $A$.

We offer the numerical parametrization of the ratios $R_{i}^{A}\left(x, Q^{2}\right)$ in two formats: The first one, called "EKS98", computes the nuclear ratios for all parton flavours within one function call, and the second one, called "EKS98r", computes the nuclear ratio of the parton flavour specified by the user. Both versions are initialized by reading in two small tables. The codes and the tables are contained in a package now available from any of the authors via email. For an easier access, the package is also placed in the WWW.

Here we do not wish to go into the numerical details of making such a parametrization. Instead, we simply demonstrate the quality of our parametrization in Figs. (11) and (2). As

\footnotetext{
${ }^{7}$ Also these tables with an interpolation routine is available from us via email.

${ }^{8}$ http://fpaxp1.usc.es/phenom/
} 
can be seen in the figures, the parametrization is based on the results [10 obtained with the set GRV-LO. Therefore the parametrization is closer to those results and the region of validity is $10^{-6} \leq x \leq 1$ and $Q^{2} \geq 2.25 \mathrm{GeV}^{2}$. For $A \leq 2$, the parametrization simply returns unity, in accordance with the fact that nuclear effects in deuterium were neglected.

Now, with the results of the previous section on the approximate set-independence of the ratios $R_{i}^{A}$, the absolute parton distributions in protons of an arbitrary nucleus $A$ with $Z$ protons and $A-Z$ neutrons can be obtained simply by multiplying the parton distributions $f_{i}^{\text {PDset }}$ of any modern lowest order set by our parametrization $R_{i}^{A \text {,EKS98 }}$,

$$
f_{i / A}^{\mathrm{PDset}}\left(x, Q^{2}\right) \equiv f_{i / p / A}^{\mathrm{PDset}}\left(x, Q^{2}\right) \approx R_{i}^{A, \mathrm{EKS} 98}\left(x, Q^{2}\right) f_{i}^{\mathrm{PDset}}\left(x, Q^{2}\right),
$$

and the corresponding distributions in a bound neutron can be obtained through the assumed isospin symmetry (exact for isoscalars, though). Thus, our parametrization should be easy to use together with parton distributions from e.g. the CERN PDFLIB [32] or other similar packages.

\section{The set-dependence in the physical quantities}

As the last task, we check explicitly the set-dependence in the physical quantities studied in Ref. [10], and simultaneously we show the range of uncertainty when using our parametrization with different sets of parton distributions in the free proton instead of the nuclear ratios obtained specifically for the chosen set. For brevity, let us call the latter ones as GRV-ratios and CTEQ-ratios.

First, in Fig. 3, we calculate the $Q^{2}$ dependence of the ratio $F_{2}^{A} / F_{2}^{\mathrm{C}}=R_{F_{2}}^{A} / R_{F_{2}}^{\mathrm{C}}$ for an isoscalar $A=118$, corresponding to an isoscalar tin, and compare our calculation with the NMC data [8]. There are four curves in the figure: two curves which have been obtained by using the nuclear parton distributions obtained with the GRV-ratios and CTEQ-ratios, and two curves obtained with our numerical parametrization. The four curves practically lie on top of each other, so the uncertainty due to the choice of the set, and the uncertainty in our parametrization, are negligible as compared to the experimental (statistical) error bars. We have checked that we arrive in the same conclusion also for other ratios $R_{F_{2}}^{A}\left(x, Q^{2}\right)$, as expected due to the small differencies of the ratios in Fig. 11.

Another quantity we study here is the ratio of the lowest order Drell-Yan cross section in $p A$ and $p \mathrm{D}$ and its comparison to the E772-data [24]. As presented in [10], the cross section ratio for a nucleus $A$ with $Z$ protons and $A-Z$ neutrons can be written as

$$
\begin{aligned}
& R_{D Y}^{A}\left(x_{2}, Q^{2}\right) \equiv \frac{\frac{1}{A} d \sigma_{D Y}^{p A} / d x_{2} d Q^{2}}{\frac{1}{2} d \sigma_{D Y}^{p D} / d x_{2} d Q^{2}} \\
& =\left\{4\left[u_{1}\left(\bar{u}_{2}^{A}+\bar{d}_{2}^{A}\right)+\bar{u}_{1}\left(u_{2}^{A}+d_{2}^{A}\right)\right]+\left[d_{1}\left(\bar{d}_{2}^{A}+\bar{u}_{2}^{A}\right)+\bar{d}_{1}\left(d_{2}^{A}+u_{2}^{A}\right)\right]+4 s_{1} s_{2}^{A}+\ldots\right\} / N_{D Y} \\
& +\left(\frac{2 Z}{A}-1\right)\left\{4\left[u_{1}\left(\bar{u}_{2}^{A}-\bar{d}_{2}^{A}\right)+\bar{u}_{1}\left(u_{2}^{A}-d_{2}^{A}\right)\right]+\left[d_{1}\left(\bar{d}_{2}^{A}-\bar{u}_{2}^{A}\right)+\bar{d}_{1}\left(d_{2}^{A}-u_{2}^{A}\right)\right]\right\} / N_{D Y}
\end{aligned}
$$

where the denominator is

$$
N_{D Y}=4\left[u_{1}\left(\bar{u}_{2}+\bar{d}_{2}\right)+\bar{u}_{1}\left(u_{2}+d_{2}\right)\right]+\left[d_{1}\left(\bar{d}_{2}+\bar{u}_{2}\right)+\bar{d}_{1}\left(d_{2}+u_{2}\right)\right]+4 s_{1} s_{2}+\ldots
$$


and where we have used the notation $q_{i}^{(A)} \equiv q_{(A)}\left(x_{i}, Q^{2}\right)$ for $i=1,2$ and $q=u, d, s, \ldots$. The scale in the parton distributions is the invariant mass $Q^{2}$ of the lepton pair. The target (projectile) momentum fraction is $x_{2}\left(x_{1}\right)$, and $x_{1}=Q^{2} /\left(s x_{2}\right)$.

In Fig. 4 , we plot the ratio $R_{D Y}^{A}$ for ${ }_{6}^{12} \mathrm{C},{ }_{20}^{40} \mathrm{Ca},{ }_{26}^{56} \mathrm{Fe}$ and ${ }_{74}^{184} \mathrm{~W}$ as a function of the target momentum fraction $x_{2}$. As in Fig. 3, we show four different calculation in each panel, corresponding to $x_{2}$ and $\left\langle Q^{2}\right\rangle$ of the data [33]: two of them are obtained by using the GRV-ratios and the CTEQ-ratios with the corresponding sets for the free proton, and the remaining two by using our numerical parametrization together with the GRV-LO and CTEQ4L parton distributions for the free proton. Carbon and calcium are isoscalar nuclei, $A=2 Z$, so for them the latter part in Eq. (8) drops out. The remaining small difference in the calculated results (the big circles and the diamonds) is then the uncertainty in our parametrization due to the set-dependence. With iron and especially with tungsten the situation becomes more interesting because the non-isoscalar effects start to play a visible role. In the panel for tungsten, we clearly see that in the calculated quantities, there are separate "error bands" for the results obtained with GRV-LO and CTEQ4L distributions. The "width" of these bands is again the uncertainty related to using our parametrization instead of the "exact" GRV - or CTEQ-ratios. As compared to the differencies in the free proton distributions and to the experimental error bars for $R_{D Y}^{A}$, the uncertainty due to using our parametrization instead of the "exact" nuclear ratios is small.

\section{Discussion and conclusions}

By using the approach of Ref. [10], we have studied the leading twist lowest order DGLAP evolution of nuclear parton densities. The nuclear effects to the initial parton densities for free proton at $Q^{2}=Q_{0}^{2}$ have been determined by using the data from the deeply inelastic $l A$ scatterings [2]- [7] and from the Drell-Yan measurements in $p A$ collisions 24], and conservation of baryon number and momentum as constraints. The nuclear parton distributions, $f_{i / A}^{\text {PDset }}\left(x, Q^{2}\right)$, obtained with different sets may differ considerably in absolute magnitude but in the scale dependent the nuclear ratios, $R_{i}^{A}\left(x, Q^{2}\right)=f_{i / A}^{\text {PDset }}\left(x, Q^{2}\right) / f_{i}^{\text {PDset }}\left(x, Q^{2}\right)$ the differencies between the sets are much smaller. By using the GRV-LO 25] and CTEQ4L [26] distributions, we have verified that the set-dependence in $R_{i}^{A}\left(x, Q^{2}\right)$ for individual parton flavours is negligible as compared to the current theoretical overall accuracy in fixing the initial nuclear effects at $Q^{2}=Q_{0}^{2}$, and also compared to the precision of the experimental data.

With the result that the dependence of the nuclear ratios on the choice for the parton distributions for the free proton is negligible, we have prepared a numerical parametrization of the ratios $R_{i}^{A}\left(x, Q^{2}\right)$ to be used for any practical applications of computing cross sections of hard scatterings in nuclear collisions, where absolute nuclear parton distributions, $f_{i / A}^{\text {PDset }}\left(x, Q^{2}\right)=R_{i}^{A}\left(x, Q^{2}\right) f_{i}^{\text {PDset }}\left(x, Q^{2}\right)$ are needed. We distribute our parametrization in a package containing two alternative Fortran routines. The package is now available from us via email or from the WWW"

In Ref. [10] it was shown that the pure leading twist, lowest order DGLAP evolution can

\footnotetext{
${ }^{9}$ http://fpaxp1.usc.es/phenom/
} 
account very well for the observed scale dependence of the structure function ratio $F_{2}^{\mathrm{Sn}} / F_{2}^{\mathrm{C}}$. Here we have demonstrated by using the GRV-LO and CTEQ4L distributions that using any modern LO set of parton distributions for the free proton will result in the same conclusion.

We have also shown that quite different isospin effects can be expected in the ratios of Drell-Yan cross sections in $p A$ vs. $p \mathrm{D}$ for large non-isoscalar nuclei like tungsten when using sets with different relative magnitudes for $\bar{u}$ and $\bar{d}$. We have also demonstrated that it is again sufficient to use set-independent nuclear ratios, i.e. our parametrization, in the computation of the DY ratios, the differencies result from the differencies between the parton distributions of the free proton.

We conclude by recalling the questions in our approach that are still open regarding the determination of the initial distributions at $Q^{2}=Q_{0}^{2}$.

At large values of $x$ the existence of the EMC-effect in the sea and for the gluon distributions is an assumption. To directly verify this experimentally may be quite difficult regarding the gluons. For the sea quarks, however, there may be a chance to have further constraints from the NA50 data for Drell-Yan in $\mathrm{Pb}-\mathrm{Pb}$ collisions at $\sqrt{s}=17.2 \mathrm{GeV}$ at the CERN-SPS, provided that the precision in the invariant mass distribution will be sufficient at large masses. If the sea quarks show an EMC effect, this should serve as an additional constraint for the gluons through the evolution.

The region at $x \lesssim 0.01$ involved assumptions of saturation of shadowing, and at very small values of $x$ the gluons were expected to have the same shadowing as $F_{2}^{A}$ (or the sea quarks). To pin down the nuclear gluon shadowing at small values of $x$, high precision data on the $Q^{2}$ dependence of $F_{2}^{A} / F_{2}^{\mathrm{D}}, F_{2}^{A} / F_{2}^{\mathrm{C}}$ would be needed especially at $x \gtrsim 0.01$, where the gluons start to determine the evolution of the sea quarks more strongly [14, 10. In the future, perhaps also the gluon initiated processes like open charm and $J / \Psi$ production in DIS 34 and in $\mathrm{p} A$ collisions [35, 36] could help in constraining the gluon antishadowing peak further.

Related to the small- $x$ physics, the role of higher twist effects like the GLRMQ fusion corrections [23] needs still to be studied further together with the results from HERA. Also the NLO analysis, both in the cross sections and in the evolution, should be performed. Most importantly, however, our analysis would benefit from an inclusion of a more quantitative estimates of uncertainties and propagation of errors.

Acknowledgements. We gratefully acknowledge C. Lourenço's efforts to get this program started. We also thank V. Ruuskanen for useful discussions and comments on the manuscript. We thank N. Armesto and the Hard Probe Collaboration, especially R. Vogt, for the useful suggestions to improve our numerical parametrization. KJE also thanks the Institute of Nuclear Theory, University of Washington, Seattle, for hospitality during the Hard Probe workshop in May 1998, where the results of this work were reported. CAS is grateful to the PPE Division of CERN (ALICE group) for hospitality during September - December 1998, when part of this work was completed. CAS thanks Xunta de Galicia for financial support. This work was supported by the Academy of Finland, grant no. 42376.

\section{References}


[1] EMC collaboration, J.J. Aubert et al., Phys. Lett. B123 (1983) 275; A. Bodek et al., Phys. Rev. Lett. 50 (1983) 1431; 51 (1983) 534.

[2] NMC collaboration, P. Amaudruz et al., Nucl. Phys. B441 (1995) 3.

[3] NMC collaboration, M. Arneodo et al., Nucl. Phys. B441 (1995) 12.

[4] NMC collaboration, M. Arneodo et al., Nucl. Phys. B481 (1996) 3.

[5] J. Gomez et al., Phys. Rev. D49 (1994) 4348.

[6] E665 collaboration, M.R. Adams et al., Phys. Rev. Lett. 68 (1992) 3266.

[7] E665 collaboration, M.R. Adams et al., Z. Phys. C67 (1995) 403.

[8] NMC collaboration, M. Arneodo et al., Nucl. Phys. B481 (1996) 23.

[9] M. Arneodo, Phys. Rep. 240 (1994) 301.

[10] K.J. Eskola, V.J. Kolhinen and P.V. Ruuskanen, Preprint CERN-TH/97-345, JYFL2/98 hep-ph/9802350, submitted to Nucl. Phys. B.

[11] Yu. Dokshitzer, Sov. Phys. JETP 46 (1977) 1649; V.N. Gribov and L. N. Lipatov, Sov. Nucl. Phys. 15 (1972) 438, 675; G. Altarelli, G. Parisi, Nucl. Phys. B126 (1977) 298.

[12] Jianwei Qiu, Nucl. Phys. B291 (1987) 746.

[13] L.L. Frankfurt, M.I. Strikman and S. Liuti, Phys. Rev. Lett. 65 (1990) 1725.

[14] K.J. Eskola, Nucl. Phys. B400 (1993) 240.

[15] V. Barone et al., Z. Phys. C58 (1993) 541.

[16] K.J. Eskola, J. Qiu and X.-N. Wang, Phys. Rev. Lett. 72 (1994) 36.

[17] S. Kumano, Phys. Rev. C50 (1994) 1247. S. Kumano and M.Miyama, Phys. Lett. B378 (1996) 267; S. Kumano and M. Miyama, Proc. Workshop on Future Physics at HERA, Hamburg, Germany, Sep 1995 and Feb 1996, Preprint SAGA-HE-104-96, [hepph/9607246]; M. Miyama, Proc. of Circum-Pan-Pacific Workshop on High-Energy Spin Physics '96, Kobe, Japan, Oct 1996, Preprint SAGA-HE-110-96 hep-ph/9611221]. S. Kumano and K. Umekawa, Preprint SAGA-HE-130-98, Mar 1998, hep-ph/9803359.

[18] S. Liuti and R. Vogt Phys. Rev. C51 (1995) 2244.

[19] D. Indumathi and Wei Zhu, Z. Phys. C74 (1997) 119; D. Indumathi, Z.Phys. C76 (1997) 91; D. Indumathi and Wei Zhu, Proc. Workshop on Future Physics at HERA, Hamburg, Sep 1995, preprint DO-TH-96-12, Sep 1995, hep-ph/9609362.

[20] Z. Huang, H.J. Lu and I. Sarcevic, Preprint AZPH-TH-97-07, May 1997, [hep$\mathrm{ph} / 9705250$ 
[21] N. Armesto and M.A. Braun, Z. Phys. C76 (1997) 81.

[22] A.L. Ayala, M.B. Gay Ducati and E.M. Levin, Nucl. Phys. B493 (1997) 305.

[23] L.V. Gribov, E.M. Levin and M.G. Ryskin, Nucl. Phys. B188 (1981) 555; Phys. Rep. 100 (1983) 1; A.H. Mueller and J. Qiu, Nucl. Phys. B268 (1986) 427.

[24] D. M. Alde et al., Phys. Rev. Lett. 64 (1990) 2479.

[25] M. Glück, E. Reya and A. Vogt, Z. Phys. C53 (1992) 53.

[26] H.L. Lai et al., Phys. Rev. D55 (1997) 1280.

[27] B. Badełek and J. Kwieciński, Phys. Rev. D50 (1994) 4; Nucl. Phys. B370 (1992) 278.

[28] H1 Collaboration, I. Abt et al., Nucl. Phys. B407 (1993) 515; T. Ahmed et al., Nucl. Phys. B439 (1995) 471; ZEUS Collaboration, M. Derrick et al., Phys. Lett. B316 (1993) 412; Z. Phys. C65 (1995) 379.

[29] H1 Collaboration, S. Aid et al., Nucl. Phys. B470 (1996) 3.

[30] T. Gousset and H.J. Pirner, Phys. Lett. B375 (1996) 349.

[31] A. Mücklich, "The structure function ratio $F_{2}^{S n} / F_{2}^{C}$ ", in Proc. of the Workshop on Deep Inelastic Scattering and QCD, Paris, 1995.

[32] Plothow-Besch, "PDFLIB: Nucleon, Pion and Photon Parton Density Functions and $\alpha_{s}$ Calculations", User's Manual -Version 7.09, W5051 PDFLIB, 1997.07.02, CERN-PPE.

[33] P. McGaughey, private communication.

[34] P. Amaudruz et al, Nucl. Phys. B371 (1992) 553.

[35] M.J. Leitch et al, Phys. Rev. Lett. 72 (1994) 2542,

[36] E866 Collaboration, M. Leitch in the workshop of "Hard Probes of Dense Matter", INT, Univ. of Washington, Seattle, May 1998. 


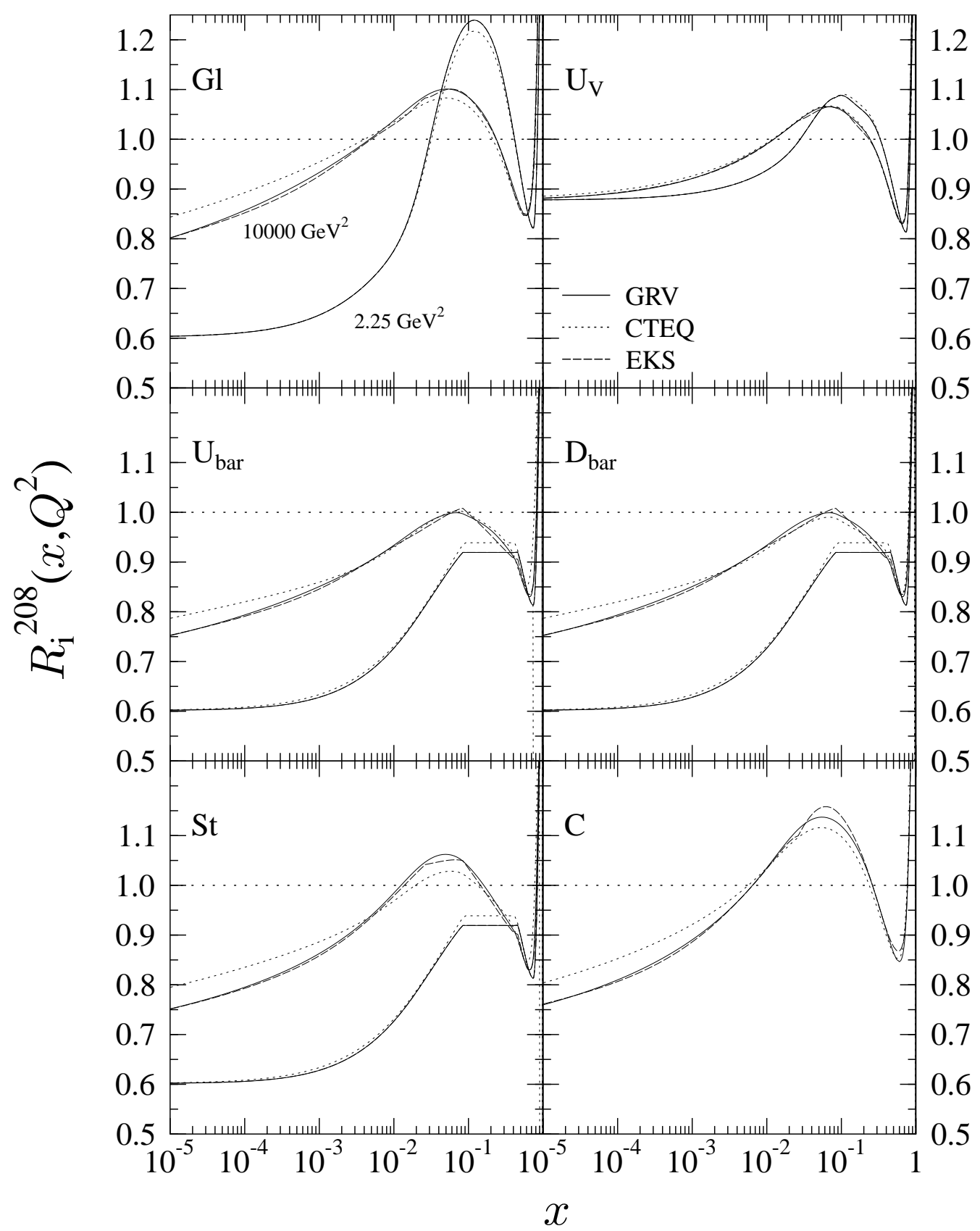

Figure 1: The nuclear ratios $R_{i}^{A}\left(x, Q^{2}\right)$ for individual parton flavours $i=g, u_{V}, \bar{u}, \bar{d}, s, c$ of a lead nucleus $A=208$ as functions of $x$ at fixed values of $Q^{2}=Q_{0}^{2}=2.25 \mathrm{GeV}^{2}$ and $Q^{2}=10000 \mathrm{GeV}^{2}$ as obtained by using the GRV-LO [25] distributions (solid lines) and the CTEQ4L [26] distributions (dotted lines) for the free proton. The dashed lines show our numerical parametrization (EKS) to the nuclear effects obtained in the GRV-LO case. The ratios $R_{d_{V}}^{A}$ are almost identical to $R_{u_{V}}^{A}$, and are not shown. The charm ratios are presented only for $Q^{2}=10000 \mathrm{GeV}^{2}$, since the charm distributions are generated only above our $Q_{0}^{2}$. The ratios $R_{b}^{A}$ at $Q^{2}=10000 \mathrm{GeV}^{2}$ behave as $R_{c}^{A}$, and are not shown. For practical purposes the set-dependence of the ratios $R_{i}^{A}\left(x, Q^{2}\right)$ is negligible. 


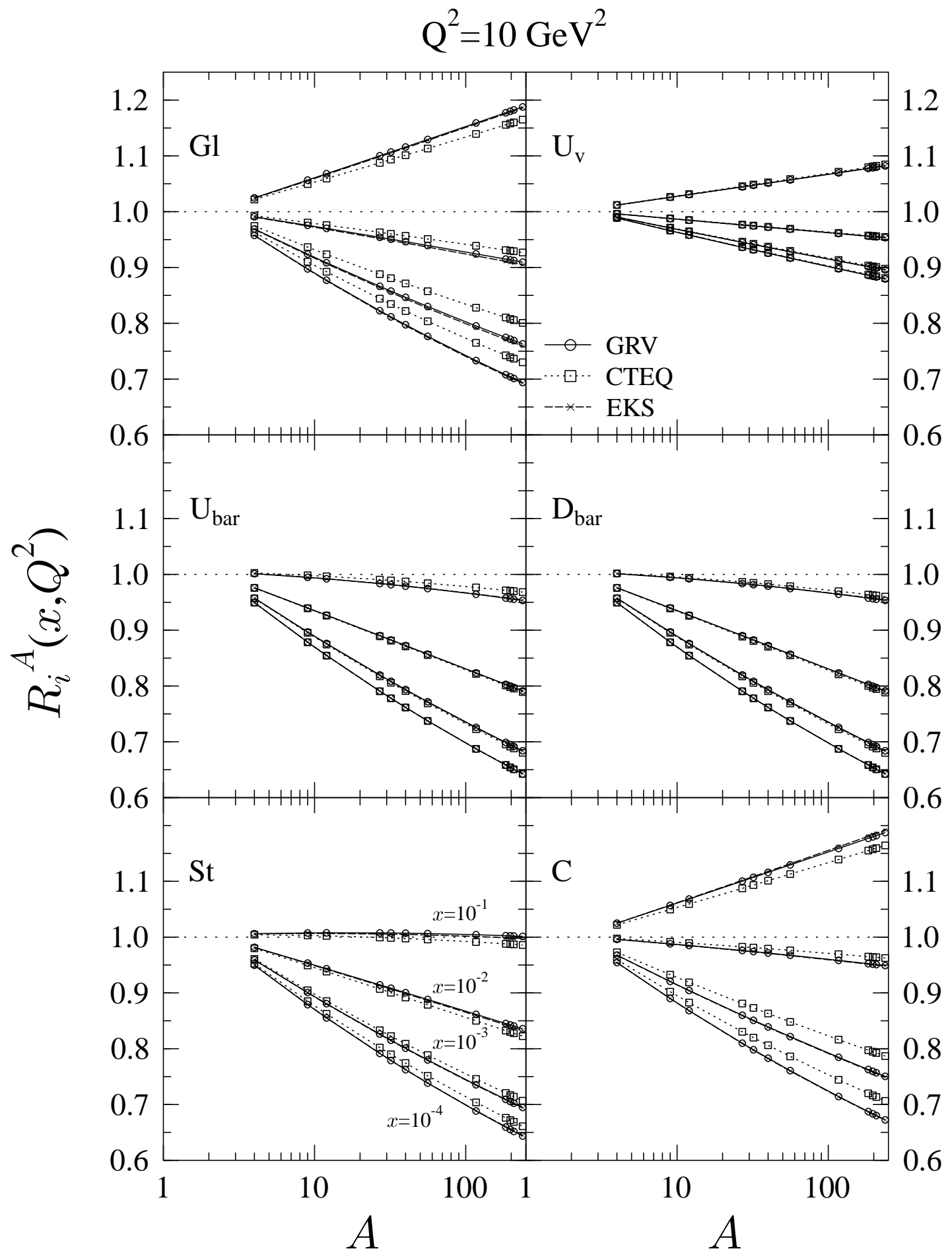

Figure 2: The nuclear ratios $R_{i}^{A}\left(x, Q^{2}\right)$ for individual parton flavours $i=g, u_{V}, \bar{u}, \bar{d}, s, c$ as functions of the mass number $A$ at a fixed value of $Q^{2}=10 \mathrm{GeV}^{2}$ and at fixed values of $x=$ $10^{-4}, 10^{-3}, 10^{-2}, 10^{-1}$ (see the panel for the strange quarks). The notation of the curves is the same as in Fig. 11 and the markers show the nuclei for which we have made the computation. The set-dependence is the largest for large nuclei but keeps within $\sim 5 \%$. 


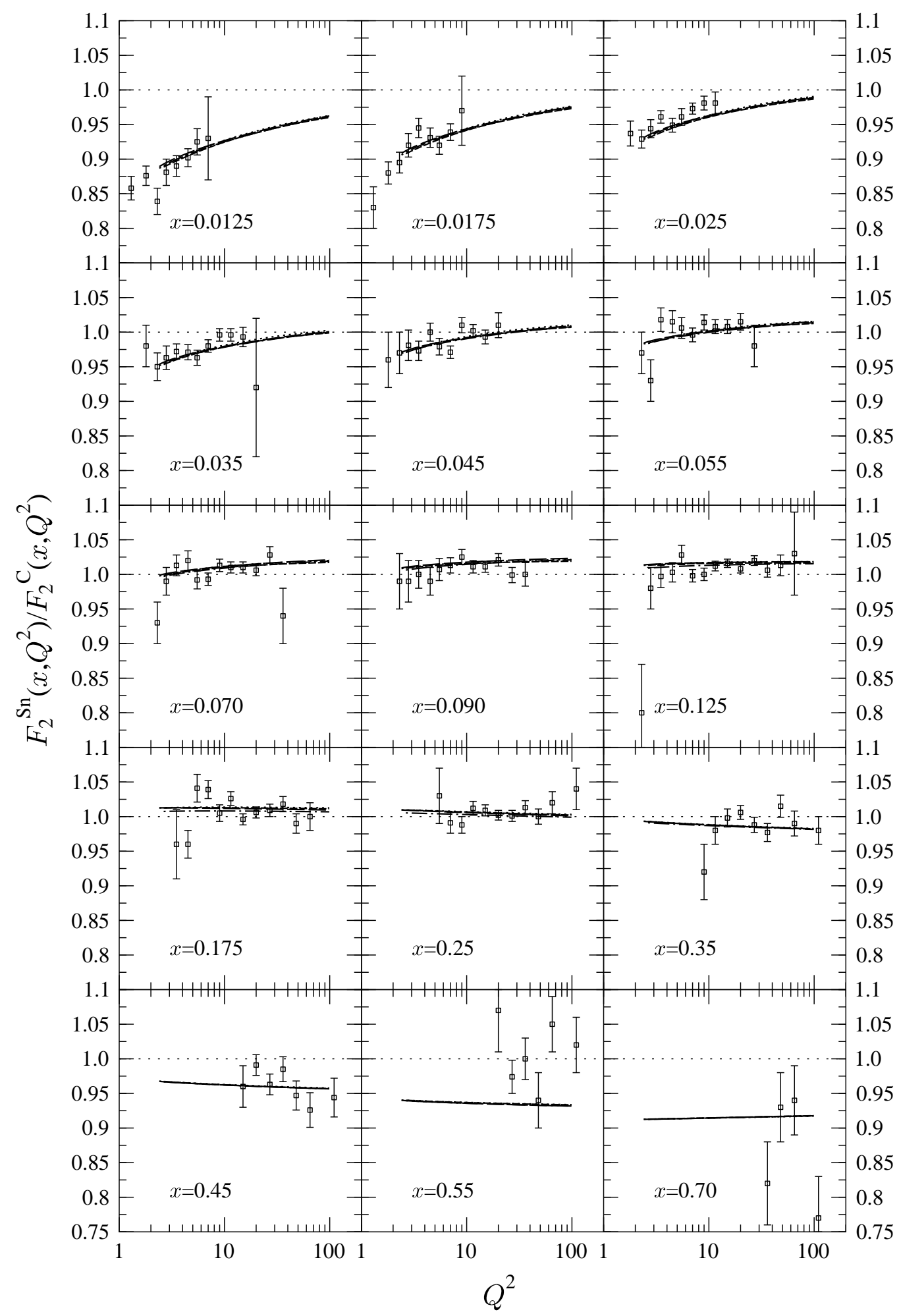

Figure 3: The ratio $F_{2}^{\mathrm{Sn}} / F_{2}^{\mathrm{C}}$ as a function of $Q^{2}$ at several different fixed values of $x$. The data is from [8]. The figure contains four calculated curves: two of the curves correspond to the "exact" results obtained with the nuclear ratios for the GRV-LO distributions (as in [10]) and for the CTEQ4L-distributions separately, and two curves correspond to the results obtained with the GRV-LO and CTEQ4L distributions multiplied by our numerical parametrization of $R_{i}^{A}\left(x, Q^{2}\right)$. There is no significant difference between the calculated curves. 


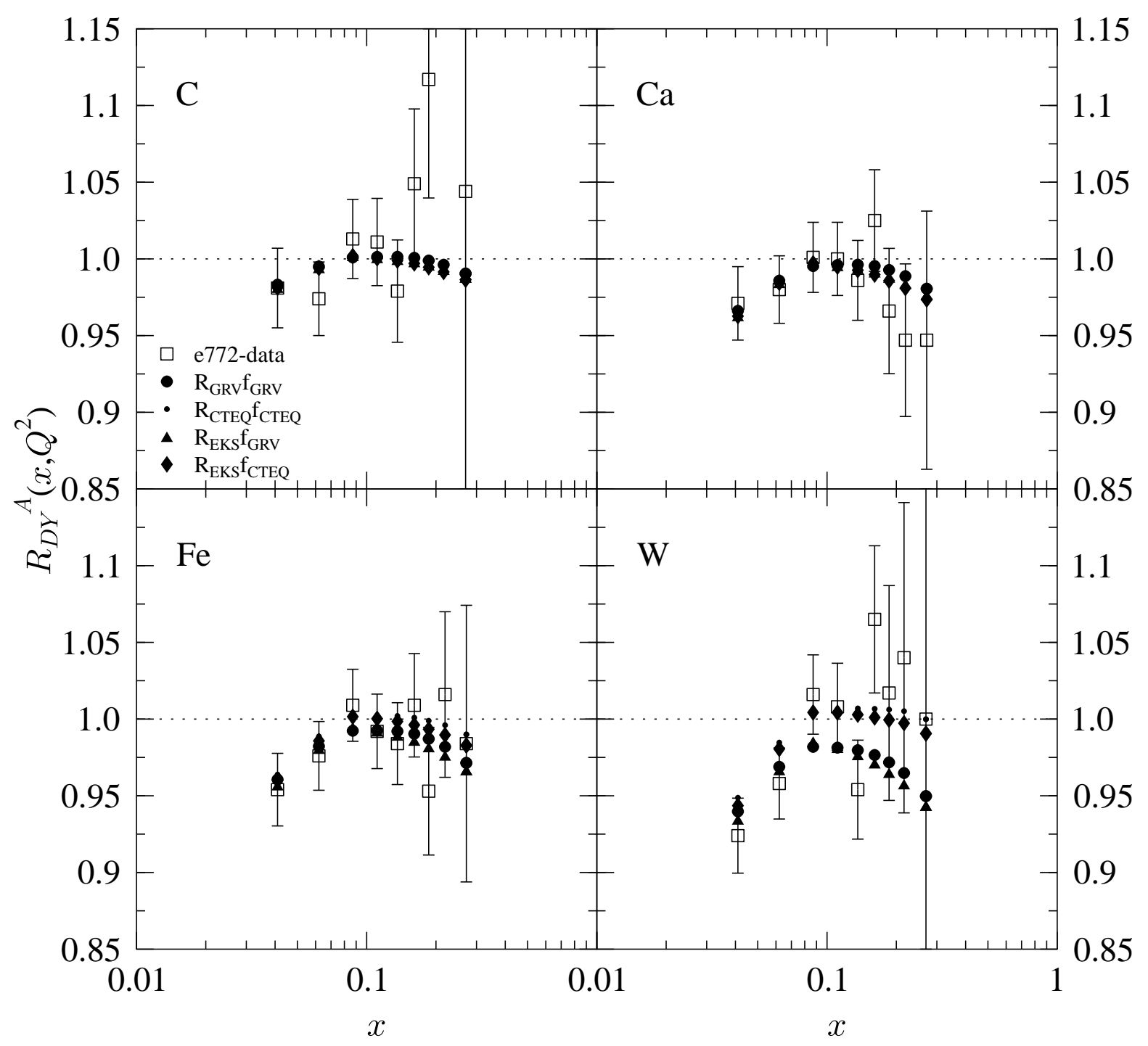

Figure 4: The ratio of differential Drell-Yan cross sections in $\mathrm{p} A$ and $\mathrm{pD}$ from Eq. (8) as a function of $x=x_{2}$ for ${ }_{6}^{12} \mathrm{C},{ }_{20}^{40} \mathrm{Ca},{ }_{26}^{56} \mathrm{Fe}$ and ${ }_{74}^{184} \mathrm{~W}$. The open squares show the E772-data [24], and the filled symbols stand for the calculated ratios $R_{D Y}^{A}\left(x, Q^{2}\right)$ at $\left(x, Q^{2}\right)$ corresponding to the experimental values [33]. The circles show $R_{D Y}^{A}$ as computed with the nuclear ratios obtained separately for the GRV-LO set (big circles) and for the CTEQ4L set (small circles). The results obtained by using our numerical parametrization (EKS) of $R_{i}^{A}$ together with the sets GRV-LO and CTEQ4L are shown by triangles and diamonds, correspondingly. As seen from the panel for tungsten, the differencies between the two parton distribution sets used for the free proton are larger than the error from using the set-independent parametrization for the nuclear effects $R_{i}^{A}$. 